\title{
Effectiveness of REGEN-COV Antibody Combination in Preventing Severe COVID-19 Outcomes
}

Samah Hayek ( $\nabla$ samahha@clalit.org.il )

Clalit Health Services

\section{Yatir Ben-Shlomo}

Clalit Research Institute

\section{Noa Dagan}

Clalit Research Institute \& Department of Biomedical Informatics, Harvard Medical School

\section{Ben Reis}

Boston Children's Hospital

\section{Noam Barda}

Clalit Research Institute https://orcid.org/0000-0002-3400-235X

\section{Eldad Kepten}

Institut Curie

\section{Alina Roitman}

Clalit Health Services

Shachar Shapira

Israel Defense Forces Medical Corps

\section{Shlomit Yaron}

Clalit Health Services

\section{Ran Balicer}

Clalit Research Institute https://orcid.org/0000-0002-7783-6362

\section{Doron Netzer}

Clalit Health Service

\section{Alon Peretz}

Clalit community Division

\section{Article}

Keywords:

Posted Date: February 18th, 2022

DOI: https://doi.org/10.21203/rs.3.rs-1338153/v1 
License: (c) (i) This work is licensed under a Creative Commons Attribution 4.0 International License. Read Full License

Version of Record: A version of this preprint was published at Nature Communications on August 2nd, 2022. See the published version at https://doi.org/10.1038/s41467-022-32253-9. 


\section{Abstract}

REGEN-COV, a combination of the monoclonal antibodies casirivimab and imdevimab, has been approved as a treatment for high-risk patients infected with SARS-CoV-2 within 5 days of their diagnosis. We used the data repositories of Israel's largest healthcare organization to determine the real-world effectiveness of REGEN-COV treatment against COVID-19 related hospitalization, severe disease, and death. We compared patients infected with the delta variant of SARS-CoV-2 and treated with REGEN-COV $(n=289)$ to those infected but untreated with REGEN-COV $(n=1,294)$. Patients were matched and further adjusted on demographic and clinical characteristics, with estimated treatment effectiveness defined as one minus the hazard ratio. Treatment effectiveness of REGEN-COV was $55.2 \%$ (95\% Cl: $21.5-74.5 \%$ ) in preventing COVID-19 hospitalization, 59.4\% (95\% Cl: 20.2-79.4\%) in preventing severe COVID-19, and 93.8\% (95\% Cl: 54.4-99.2\%) in preventing COVID-19 death in the 28 days after treatment. In conclusion, REGEN-COV was effective in reducing the risk of severe sequelae in high-risk COVID-19 patients.

\section{Introduction}

Since December 2019, SARS-CoV-2 has spread worldwide, ${ }^{1}$ resulting in over 310 million confirmed infections and over 5.4 million deaths. ${ }^{2}$ SARS-CoV-2 vaccines, first introduced in December 2020, have helped reduce the burden of the pandemic, yet there remain many people who are not vaccinated, either due to lack of vaccine access, prior medical conditions, or vaccine hesitancy. ${ }^{3}$ and breakthrough infections occur among vaccinated individuals. ${ }^{4}$ Therefore, there is still a need for effective treatments to prevent severe COVID-19 outcomes among infected individuals.

REGEN-COV, a combination of two SARS-CoV-2 antibodies (Casirivimab and Imdevimab), received emergency use authorization by the US Food and Drug Administration for patients aged 12 years or older who have been diagnosed with COVID-19 and have not deteriorated to severe illness, but are at high risk for deterioration - including immunocompromised individuals and those with high-risk medical conditions. ${ }^{5}$ The effectiveness of REGEN-COV has been demonstrated in clinical trials, ${ }^{5-7}$ where it was shown to reduce symptomatic COVID-19 illness by $62 \%$ and COVID-19-related hospitalization or death by $70 \%$ in the first month after treatment. ${ }^{5}$ Evidence suggests that the benefits are greater when REGEN-COV is administered within the first 5 days following infection..$^{8,9}$ An observational study of 696 patients who received REGEN-COV found that the treatment significantly lowered all-cause hospitalization rates at day 14 (1.3\% vs 3.3\%; absolute difference[AD]: $2.0 \%$; $95 \%$ confidence interval (CI): $0.5-3.7 \%)$ and at day 21 (1.3\% vs $4.2 \%$; AD: $2.9 \% ; 95 \% \mathrm{Cl}: 1.2-4.7 \%) .{ }^{10}$ However, this observational study reported a single nonCOVID-19 outcome and was conducted among a specific population in the US, which may limit the generalizability of its findings.

The Israeli Ministry of Health began a nationwide campaign to administer REGEN-COV at a dose of 1200 mg on September 22, 2021, during a period in which the Delta variant was the dominant variant in Israel, and Israel's fourth wave of COVID-19 was at its peak. As part of this campaign, Clalit Health Services 
(CHS), Israel's largest healthcare organization, proactively offered REGEN-COV treatment at home to eligible members who were diagnosed with COVID-19. Members were eligible if they were within 5 days of their first positive Polymerase Chain Reaction (PCR) test and were determined to be at high risk for severe illness, but had not yet developed severe COVID-19. The determination of risk was made by clinicians at the point-of-care, based on patients' current medical condition, medical history including chronicconditions, and overall likelihood of deterioration to severe illness.

The present study aims to evaluate the real-world effectiveness of REGEN-COV treatment in preventing COVID-19 related hospitalization, severe illness and death. A retrospective cohort study was conducted using the data repositories of Israel's largest healthcare organization. Patients diagnosed with COVID-19 between September 19, 2021 and December 8, 2021 who were treated with REGEN-COV were matched to patients diagnosed with COVID-19 between July 1, 2021 and December 8, 2021 who were not treated with REGEN-COV. Analysis was performed using Cox regression, with estimated treatment effectiveness defined as one minus the hazard ratio. A subgroup analysis by age group ( $<60$ or $\geq 60$ year old) was conducted as a secondary analysis.

\section{Results}

In total, 162,795 CHS members tested positive for COVID-19 between July 1 and December 8, 2021; 135,458 were eligible for the study. Of this population, 289 patients treated with REGEN-COV were matched with 1,294 untreated patients (Supplemental Fig. 1). Following matching, all matched variables were well-balanced between the treated and untreated groups (Supplemental Fig. 2). The median age of the population was 67 years (interquartile range: $58-74$ years) and $49 \%$ were men. Patients treated with REGEN-COV were more likely to be smokers compared to those who were untreated ( $15 \%$ vs. $11 \%$, respectively) (Table 1 ). 
Table 1

Baseline characteristics of the study population, by REGEN-COV status

\begin{tabular}{|c|c|c|c|}
\hline & $\begin{array}{l}\text { Total } \\
\text { participants } \\
(\mathrm{N}=1,583)\end{array}$ & $\begin{array}{l}\text { Untreated with REGEN- } \\
\text { COV } \\
(N=1,294)\end{array}$ & $\begin{array}{l}\text { Treated with REGEN- } \\
\text { COV } \\
(\mathrm{N}=289)\end{array}$ \\
\hline Age[Mean(SD), median(IQR)] & $\begin{array}{l}65(14), 67 \\
(58,74)\end{array}$ & $65(14), 67(58,74)$ & $66(14), 68(58,76)$ \\
\hline \multicolumn{4}{|l|}{ Age Group, in years } \\
\hline $19-29$ & $12(0.8 \%)$ & $10(0.8 \%)$ & $2(0.7 \%)$ \\
\hline $30-39$ & $51(3.2 \%)$ & $44(3.4 \%)$ & $7(2.4 \%)$ \\
\hline $40-49$ & $192(12 \%)$ & $158(12 \%)$ & $34(12 \%)$ \\
\hline $50-59$ & $195(12 \%)$ & $162(13 \%)$ & $33(11 \%)$ \\
\hline $60-69$ & $475(30 \%)$ & $389(30 \%)$ & $86(30 \%)$ \\
\hline $70-74$ & $266(17 \%)$ & $222(17 \%)$ & $44(15 \%)$ \\
\hline $75+$ & $392(25 \%)$ & $309(24 \%)$ & $83(29 \%)$ \\
\hline \multicolumn{4}{|l|}{ Population sector } \\
\hline General Jewish & $1,133(72 \%)$ & $928(72 \%)$ & $205(71 \%)$ \\
\hline Arab & $347(22 \%)$ & $281(22 \%)$ & $66(23 \%)$ \\
\hline Orthodox Jewish & $103(6.5 \%)$ & $85(6.6 \%)$ & $18(6.2 \%)$ \\
\hline \multicolumn{4}{|l|}{ Sex } \\
\hline Female & $819(52 \%)$ & $670(52 \%)$ & $149(52 \%)$ \\
\hline Male & $764(48 \%)$ & $624(48 \%)$ & $140(48 \%)$ \\
\hline \multicolumn{4}{|l|}{ Socioeconomic status } \\
\hline Low & $1,078(68 \%)$ & $889(69 \%)$ & $189(65 \%)$ \\
\hline Medium & $468(30 \%)$ & $382(30 \%)$ & $86(30 \%)$ \\
\hline High & $36(2.3 \%)$ & $22(1.7 \%)$ & $14(4.8 \%)$ \\
\hline Missing & $1(<0.1 \%)$ & $1(<0.1 \%)$ & $0(0 \%)$ \\
\hline $\begin{array}{l}\text { Flu vaccination in the last } 5 \\
\text { years }\end{array}$ & & & \\
\hline
\end{tabular}

Abbreviations:

IQR, interquartile range. 


\begin{tabular}{|c|c|c|c|}
\hline & $\begin{array}{l}\text { Total } \\
\text { participants } \\
(\mathrm{N}=1,583)\end{array}$ & $\begin{array}{l}\text { Untreated with REGEN- } \\
\text { COV } \\
(N=1,294)\end{array}$ & $\begin{array}{l}\text { Treated with REGEN- } \\
\text { COV } \\
(\mathrm{N}=289)\end{array}$ \\
\hline 0 & $422(27 \%)$ & 367 (28\%) & $55(19 \%)$ \\
\hline 1 & 207 (13\%) & $168(13 \%)$ & $39(13 \%)$ \\
\hline 2 & $180(11 \%)$ & $148(11 \%)$ & $32(11 \%)$ \\
\hline 3 & $165(10 \%)$ & $131(10 \%)$ & $34(12 \%)$ \\
\hline 4 & $213(13 \%)$ & $155(12 \%)$ & $58(20 \%)$ \\
\hline 5 & $396(25 \%)$ & $325(25 \%)$ & $71(25 \%)$ \\
\hline \multicolumn{4}{|l|}{ Body mass index $\left(\mathrm{kg} / \mathrm{m}^{2}\right)$} \\
\hline Underweight & $18(1.1 \%)$ & $13(1.0 \%)$ & $5(1.7 \%)$ \\
\hline Normal & $371(23 \%)$ & 305 (24\%) & $66(23 \%)$ \\
\hline Obese & $688(43 \%)$ & $562(43 \%)$ & $126(44 \%)$ \\
\hline Overweight & $506(32 \%)$ & $414(32 \%)$ & $92(32 \%)$ \\
\hline \multicolumn{4}{|l|}{ Smoking status } \\
\hline Current smoker & $184(12 \%)$ & $143(11 \%)$ & $41(14 \%)$ \\
\hline Past smoker & $393(25 \%)$ & 318 (25\%) & $75(26 \%)$ \\
\hline Non-smoker & $1,006(64 \%)$ & $833(64 \%)$ & $173(60 \%)$ \\
\hline Recent full vaccination & $1,187(75 \%)$ & $1,011(78 \%)$ & $176(61 \%)$ \\
\hline \multicolumn{4}{|l|}{ First vaccination dose } \\
\hline Unvaccinated & $513(32 \%)$ & $414(32 \%)$ & $99(34 \%)$ \\
\hline $0-3$ weeks & $692(44 \%)$ & $571(44 \%)$ & $121(42 \%)$ \\
\hline 4-7 weeks & $190(12 \%)$ & $163(13 \%)$ & $27(9.3 \%)$ \\
\hline $8-10$ weeks & $121(7.6 \%)$ & $102(7.9 \%)$ & $19(6.6 \%)$ \\
\hline $11-19$ weeks & $57(3.6 \%)$ & $39(3.0 \%)$ & $18(6.2 \%)$ \\
\hline$\geq 20$ weeks & $10(0.6 \%)$ & $5(0.4 \%)$ & $5(1.7 \%)$ \\
\hline
\end{tabular}

Abbreviations:

$I Q R$, interquartile range. 


\begin{tabular}{|llll|}
\hline & $\begin{array}{l}\text { Total } \\
\text { participants } \\
(\mathbf{N = 1 , 5 8 3 )}\end{array}$ & $\begin{array}{l}\text { Untreated with REGEN- } \\
\text { COV } \\
\mathbf{( N = 1 , 2 9 4 )}\end{array}$ & $\begin{array}{l}\text { Treated with REGEN- } \\
\text { COV }\end{array}$ \\
\hline Cancer & $84(5.3 \%)$ & $59(4.6 \%)$ & $25(8.7 \%)$ \\
\hline Chronic kidney disease & $313(20 \%)$ & $226(17 \%)$ & $87(30 \%)$ \\
\hline Respiratory diseases & $254(16.1 \%)$ & $197(15.2 \%)$ & $57(19.7 \%)$ \\
\hline Cardiovascular disease & $475(30 \%)$ & $359(27.7 \%)$ & $116(41.0 \%)$ \\
\hline Pregnancy & $11(0.7 \%)$ & $9(0.7 \%)$ & $2(0.7 \%)$ \\
\hline Diabetes & $552(34.9 \%)$ & $427(32.6 \%)$ & $125(43.1 \%)$ \\
\hline Hypertension & $731(46 \%)$ & $573(44 \%)$ & $158(55 \%)$ \\
\hline Immunosuppression & $91(5.7 \%)$ & $74(5.7 \%)$ & $38(13 \%)$ \\
\hline Neurological disease & $180(11 \%)$ & $142(11 \%)$ & $21(7.3 \%)$ \\
\hline Liver disease & $72(4.5 \%)$ & $51(3.9 \%)$ & \\
\hline Abbreviations: & & & \\
\hline IQR, interquartile range. & & & \\
\hline
\end{tabular}

Among those treated with REGEN-COV, the risk of hospitalization due to COVID-19 decreased by $55.2 \%$ (95\% Cl: $21.5-74.5 \%)$, the risk of severe COVID-19 illness decreased by 59.4\% (95\% Cl: 20.2-79.4\%), and the risk of COVID-19-related death decreased by $93.8 \%$ (95\% Cl: 54.4-99.2\%) (Table 2). 
Outcomes associated with REGEN-COV treatment effectiveness

\section{REGEN-COV effectiveness}

\section{$(95 \% \mathrm{Cl})$}

Hospitalization due to COVID-19

Severe COVID-19
$55.2 \%(21.5 \%-74.5 \%)$

$59.4 \%(20.2 \%-79.4 \%)$

$93.8 \%(54.4 \%-99.2 \%)$

Death due to COVID-19

Note: Treatment effectiveness was measured as 1-Hazard ratio (HR), derived from the adjusted cox proportional model that was applied after the matching. Patients were matched for: Age, population sector, sex, SES, BMI, immunosuppression status, pregnancy, and first vaccination dose status (vaccinated vs. unvaccinated; those who were vaccinated were treated as the number of weeks from the beginning of the COVID-19 national vaccination campaign until the first vaccination as an indication for health seeking behavior). The model was then further adjusted for: Age, population sector, sex, SES, BMI, number of flu vaccines received in the 5 years prior to COVID-19 infection, smoking status, number of vaccine doses, recent full vaccination status, first vaccination dose, and chronic diseases (cancer, chronic kidney disease, respiratory diseases, cardiovascular diseases, diabetes, hypertension, immunosuppression, neurological conditions, and liver diseases). Complete variable definitions are found in Supplemental Table 1.

Abbreviation: $\mathrm{Cl}$, confidence interval

The results of the secondary analysis showed that among those aged 60 years or older and treated with REGEN-COV, the risk of hospitalization due to COVID-19 decreased by 55.3\% (95\% Cl: $16.0-75.7 \%$ ), the risk of severe COVID-19 illness decreased by $61.5 \%$ (95\% Cl: 20.8-81.3\%), and the risk of COVID-19-related death decreased by $95.0 \%$ (95\% Cl: 62.8-99.3\%). Among those younger than 60 years old, the risk of hospitalization due to COVID-19 decreased by $91.5 \%$ (95\% Cl: $28.2-99.0 \%)$. However, due to the rarity of severe COVID-19 and death in this age group, the effectiveness of REGEN-COV for these outcomes could not be accurately estimated (Supplemental table 1).

\section{Discussion}

In the current study, we estimated the effectiveness of community-based REGEN-COV treatment for patients newly infected with SARS-CoV-2 (Delta variant) who were determined to be at high risk for severe COVID-19, but who had not yet developed severe disease. Our results indicate that treatment with REGENCOV was effective in reducing the risk of hospitalization due to COVID-19, severe COVID-19, and COVID19-related death among patients overall and specifically for those aged 60 years or older.

The results of this real-world study are consistent with the results of the phase-III clinical trial, which showed that treatment with REGEN-COV reduced the risk of hospitalization or death by $70.4 \%$ in the 28 days following treatment initiation. ${ }^{11}$ They are also consistent with results of an observational study that showed a 70\% reduction in the need for further treatment among those treated with REGEN-COV. ${ }^{9}$ Importantly, the effectiveness of REGEN-COV has recently been reported to be diminished against the Omicron variant, ${ }^{12}$ which has become dominant in many regions worldwide. 
The current study had several limitations. First, despite the careful matching of treated and untreated individuals, there exists the possibility of residual confounding, specifically by behavioral factors that were not well-captured in our data. Second, the recruitment of the untreated patients started 10-weeks earlier than the treated patients, which could result in confounding by calendar time. However, the circumstances in Israel were not different between the first 10-weeks and the remaining study period, so we do not expect this to result in substantial bias. Third, there is the possibility of selection bias among those treated with REGEN-COV compared to those who refused to be treated with REGEN-COV and were included as controls. However, only a subset of the control population is composed of individuals who refused treatment, which limits the potential bias.

A strength of the study is that it is based on a national treatment campaign in which treatment was offered equally to all eligible patients. Furthermore, data in this study is based on a large representative cohort that covers over half of the Israeli population.

In conclusion, this study provides evidence for the effectiveness of REGEN-COV in treating high-risk COVID-19 patients recently infected with the Delta variant. With many individuals remaining unvaccinated, and breakthrough infections occurring among the vaccinated, effective treatment for COVID-19 remains vital.

\section{Methods}

\section{Data Sources}

Data were extracted from the CHS database. CHS is the largest integrated payer-provider healthcare organization in Israel. The CHS database contains extensive medical histories of CHS's 4.7 million members, including COVID-19 test results and outcomes. These data repositories have been previously described in detail. ${ }^{13,14}$

\section{Study design and population}

We conducted a cohort study using CHS data to estimate the real-world effectiveness of REGEN-COV in preventing severe COVID-19-related outcomes. Eligibility criteria included: A documented first positive SARS-CoV-2 polymerase-chain-reaction (PCR) test result; a determination of being high-risk for severe COVID-19 based on medical history and clinical characteristics; age 12 years or older; and at least one year of continuous CHS membership as of the infection date. We excluded patients who were known to be infected with the omicron variant (based on sequencing of viral samples or on the S-gene target failure (SGTF) technique; the prevalence of omicron during the study period was negligible). We also excluded patients with invalid outcome data and those who received a positive PCR result during a hospitalization for another condition.

To emulate a target trial, treated patients were individually matched with untreated patients. Treated patients were those with a first positive PCR test result between September 19, 2021 and December 8, 
2021 who received REGEN-COV treatment; Untreated patients were those with a first positive PCR test result between July 1, 2021 and December 8, 2021 who did not receive REGEN-COV treatment. The recruitment period for the untreated patients was a few weeks longer than for the treated patients to increase the sample size of the untreated group and allow for 1:5 matching of treated to untreated individuals.

REGEN-COV was not administered to certain high-risk individuals for a range of possible reasons, including: The patient was diagnosed before REGEN-COV was being offered by the healthcare system, logistic complexity prevented distribution of the treatment to the patient's home, or the patient refused to receive the treatment. The index date for the treated patients was defined as the date of REGEN-COV treatment. Untreated matched patients were given an index date based on the time from infection diagnosis to treatment of the matched treated patient: e.g., if the treated patient received REGEN-COV two days after their positive PCR test result, the index date for the matched untreated patient was set to 2 days after their positive PCR test result.

\section{Outcomes and Follow-up}

Three outcomes were examined: COVID-19 related hospitalization, severe COVID-19 illness, and death due to COVID-19. The treated and untreated patients were followed until the occurrence of the outcome or until 28-days from the index date.

\section{Covariates}

Adjustment was performed in two phases. First, the treated and untreated patients were matched on an initial set of confounders. Then, further adjustment was performed with a regression model. Subjects were matched on: Age, population sector (Jewish, Arab, Ultra-Orthodox), sex, socioeconomic status (SES, based on place of residence and categorized into 20 levels), body mass index (BMI, as a continuous variable), immunosuppression status, pregnancy, and calendar week of first vaccination dose. Confounders that were adjusted for in the model included: Age, sex, population sector, SES (as above), number of flu vaccines received within the 5 years prior to COVID-19 diagnosis, BMI (as a categorical variable: underweight, normal and obese), smoking status, number of COVID-19 vaccination doses received, "recent full vaccination" status, and calendar week of first vaccination dose. We also adjusted for the presence of the chronic conditions described in Supplemental Table 1. We included again in the regression model some of the variables were matched for to better control possible residual confounding, as mentioned in the statistical analysis. All variables were extracted according to the most recently documented value before the positive testing date, as recorded in the patients' medical records. Full variable definitions are presented in Supplemental Table 2.

Matched untreated individuals who experienced an outcome between their positive PCR test date and their assigned index date were excluded. Because the index date was only set after matching (based on the timing the matched treated counterpart was treated), this exclusion could only happen after matching. 


\section{Statistical analysis}

Matching was performed at a ratio of 1:5 treated to untreated individuals, using an optimal matching scheme. The Mahalanobis distance metric was used for continuous variables, and exact matching was used for categorical variables. ${ }^{15,16}$ Optimal matching minimizes the overall pair-wise distances without dependency on the order of matching.

After the matching, Cox proportional hazards models were fit for each outcome, adjusting for the abovementioned potential confounders. We report one minus the hazard ratio (HR) with $95 \%$ confidence intervals as the measure of treatment effect. Adjustment was performed using both matching and Cox modeling for two reasons: First, some of the variables were continuous and the matching was not exact. Second, not all the treated subjects had the same number of controls, due to the exclusion of controls post-matching. Missing data are rare in CHS database for the variables used, thus we used complete case analysis.

A subgroup analysis by age group ( $<60$ or $\geq 60$ year old) was conducted as a secondary analysis.

\section{Ethics}

This study was approved by the CHS Institutional Review Board.

\section{Declarations}

Data availability: Due to national and organizational data privacy regulations, individual-level data such as those used for this study cannot be shared.

Acknowledgements: This study was supported by the Ivan and Francesca Berkowitz Family Living Laboratory Collaboration at Harvard Medical School and Clalit Research Institute

Author contributions: R.D.B. D.N. A.P contributed equally as senior authors to this study. S.H., Y.B.S., N.D., B.Y.R., N.B., E.K., and R.D.B. conceived and designed the study. Y.B.S., N.D., E.K., N.B., participated in data extraction and analysis. S.H., Y.B.S., N.D., B.Y.R., N.B., E.K., I.L, S.S, S.Y., R.D.B, D.N., A.P. wrote the manuscript. S.S., S.Y., D.N, A.P, provided clinical guidance. All authors critically reviewed the manuscript and decided to proceed with publication. R.D.B. vouches for the data and analysis.

Competing interests: The authors have no conflicts of interest to declare

\section{References}

1. Zhu N, Zhang D, Wang W, et al. A Novel Coronavirus from Patients with Pneumonia in China, 2019. The New England journal of medicine. 2020;382(8):727-733.

2. Medicine JHU. COVID-19 DashBoard 2022; https://gisanddata.maps.arcgis.com/apps/dashboards/bda7594740fd40299423467b48e9ecf6. 
Accessed January 12, 2022.

3. Denford S, Mowbray F, Towler L, et al. Exploration of attitudes regarding uptake of COVID-19 vaccines among vaccine hesitant adults in the UK: A qualitative analysis. medRxiv. 2021:2021.2012.2023.21268325.

4. Bergwerk M, Gonen T, Lustig Y, et al. Covid-19 Breakthrough Infections in Vaccinated Health Care Workers. New England Journal of Medicine. 2021;385(16):1474-1484.

5. FDA. FDA authorizes REGEN-COV monoclonal antibody therapy for post-exposure prophylaxis (prevention) for COVID-19. 2021 https://www.fda.gov/drugs/drug-safety-and-availability/fdaauthorizes-regen-cov-monoclonal-antibody-therapy-post-exposure-prophylaxis-prevention-covid-19. Accessed 07/10/2021, 2021

6. O’Brien MP, Forleo-Neto E, Musser BJ, et al. Subcutaneous REGEN-COV Antibody Combination for Covid-19 Prevention. medRxiv. 2021:2021.2006.2014.21258567.

7. Weinreich DM, Sivapalasingam S, Norton T, et al. REGEN-COV Antibody Combination and Outcomes in Outpatients with Covid-19. New England Journal of Medicine. 2021.

8. Verderese JP, Stepanova M, Lam B, et al. Neutralizing Monoclonal Antibody Treatment Reduces Hospitalization for Mild and Moderate Coronavirus Disease 2019 (COVID-19): A Real-World Experience. Clinical Infectious Diseases. 2021.

9. Kakinoki Y, Yamada K, Tanino Y, et al. Impact of Antibody Cocktail Therapy Combined with Casirivimab and Imdevimab on Clinical Outcome for Covid-19 patients in A Real-Life Setting: A Single Institute Analysis. medRxiv. 2021.

10. Razonable RR, Pawlowski C, O'Horo JC, et al. Casirivimab-Imdevimab treatment is associated with reduced rates of hospitalization among high-risk patients with mild to moderate coronavirus disease19. EClinicalMedicine. 2021;40:101102.

11. Weinreich DM, Sivapalasingam S, Norton T, et al. REGEN-COV Antibody Cocktail in Outpatients with Covid-19. medRxiv. 2021.

12. REGENERON https://investor.regeneron.com/static-files/4aed42a1-3d26-48af-bd01-3f0c92938c11. Accessed January 2022.

13. Barda N, Dagan N, Ben-Shlomo Y, et al. Safety of the BNT162b2 mRNA Covid-19 vaccine in a nationwide setting. New England Journal of Medicine. 2021;385(12):1078-1090.

14. Dagan N, Barda N, Kepten E, et al. BNT162b2 mRNA Covid-19 Vaccine in a Nationwide Mass Vaccination Setting. New England Journal of Medicine. 2021;384(15):1412-1423.

15. Daniel Ho KI, Gary King, Elizabeth Stuart, Noah Greifer. Matchlt. 2021 https://kosukeimai.github.io/Matchlt/reference/method_optimal.html. Accessed November 302021.

16. Stuart EA, King G, Imai K, Ho D. Matchlt: nonparametric preprocessing for parametric causal inference. Journal of statistical software. 2011.

\section{Supplementary Files}


This is a list of supplementary files associated with this preprint. Click to download.

- SupplementalTablesandFigures10022022.docx 